\title{
Changes in the thermal stability of 2G HTS wires by local modification of the stabilization layer
}

\author{
Luis A. Angurel, Elena Martínez, Jorge Pelegrín, Ruth Lahoz, Germán F. de la Fuente, Nieves Andrés, \\ M. Pilar Arroyo, Yi-Yuan Xie, and Venkat Selvamanickam
}

\begin{abstract}
In order to use 2G HTS wires in superconducting devices, it is important to understand the origin of thermal instabilities in these materials. Previous results showed that inhomogeneities in the critical current distribution are not the only factor that determines the heat generation distribution along the sample when the current exceeds the critical value. In this work, different types of controlled defects have been generated in the $\mathrm{Cu}$-layer using laser ablation. In all cases, laser parameters have been chosen while assuring that the critical current of the superconductor does not deteriorate. It has been obtained that even if the thickness of the stabilizing layer is reduced from 20 $\mu \mathrm{m}$ to less than $10 \mu \mathrm{m}$ in some regions, the sample response to overcurrents is not modified.
\end{abstract}

Index Terms-Coated conductors, Thermal stability, Laser Ablation

\section{INTRODUCTION}

$\mathrm{T}$ $\mathrm{HE}$ understanding of the mechanisms that are responsible of the instabilities that take place in technological second generation high temperature superconductor (2G HTS) wires is important in order to establish which are the limits of these materials in different applications. Obviously, inhomogeneities in the critical current $\left(I_{\mathrm{c}}\right)$ distribution along the sample are one of the possible factors that can affect the response of the material. But this is not the only one. Measurements in samples with induced controlled defects in the superconducting layer [1] showed that the origin of the hot spot was not always located in the region with the lowest $I_{c}$ value. For different cooling conditions, the hot spot is originated in different places.

Manuscript received 3 August 2010. This work was supported in part by the Spanish Ministry of Science and Innovation (projects MAT2008-05983C03-01 and -03, CEN2007-2014) and by the Gobierno de Aragón (T12, T74 and $\mathrm{T} 63$ research groups).

L.A. Angurel, E. Martínez, J. Pelegrín, R. Lahoz and G.F. de la Fuente are with the Instituto de Ciencia de Materiales de Aragón (CSIC-Universidad de Zaragoza), 50018 Zaragoza, Spain (corresponding author to provide phone: 34976762520; fax: 34976761957; e-mail: angurel@unizar.es).

N. Andrés and M.P. Arroyo are with the Instituto de Investigación en Ingeniería de Aragón, Universidad de Zaragoza, 50009 Zaragoza, Spain (email:nandres@unizar.es, arroyo@unizar.es).

Y.-Y. Xie is with SuperPower, Inc., 450 Duane Ave., Schenectady, NY 12304, USA (e-mail: yxie@superpower-inc.com).

V. Selvamanickam is with the University of Houston, 4800 Calhoun Road, Texas 77004, USA, on leave from SuperPower, Inc., 450 Duane Ave., Schenectady, NY 12304, USA (e-mail: selva@superpower-inc.com).
Due to the microstructure of these materials, the stabilizing layer also plays an important role in their thermal stability response. Some studies have analyzed the influence of different stabilizers [2] or the effect of the stabilizer thickness [3] on quench development and propagation. In a great number of experimental works, usually a normal zone region is initiated using a heater fixed on the sample surface. Other works have also proposed models to investigate the influence of the superconductor-stabilizer interfacial resistance $[4,5]$ on quench propagation. These studies have shown that the stabilizing layer plays an important role in the minimum quench energy and normal zone propagation velocity values. It is still an open question whether the heat generation along the sample would be homogeneous or not when the current exceed $I_{c}$, and which are the factors that determine the existence of inhomogeneities in the heat generation which may give place to hot spots.

In this work, the possible influence of defects in the stabilizing layer in the response of the material to overcurrents has been investigated.

\section{EXPERIMENTAL PROCEDURE}

Experiments were performed on 4mm-wide 2G HTS SCS4050 wires fabricated by SuperPower, Inc.. These materials were stabilized by electroplating a $20 \mu \mathrm{m} \mathrm{Cu}$ layer on all sides of the conductor. The sample was mounted in a system prepared to perform simultaneous transport, thermal and optical characterizations [6, 7]. Four voltage taps and three thermocouples were soldered to the sample in order to characterize the evolution of the sample in three regions of 1.5, 1.4 and $1.4 \mathrm{~cm}$-long (Fig. 1(a)). All these connections were soldered at the beginning of the characterization process and were not modified until all the experiments were performed in order to assure that any possible change is not associated with modifications in the position of the voltage taps. Initially the $I-V$ curve and the time evolution of the electric field and temperature along the sample subjected to overcurrents were recorded with the sample immersed in liquid nitrogen.

Then, a laser ablation technique was used to induce some defects in the $\mathrm{Cu}$ stabilizing layer. They were produced using a Q-switched Nd:YAG PowerLine-E (Rofin-Sinar) laser, that generated a pulsed radiation with a wavelength of $1064 \mathrm{~nm}$ and a pulse width in the range of 10-50 ns. Laser parameters 


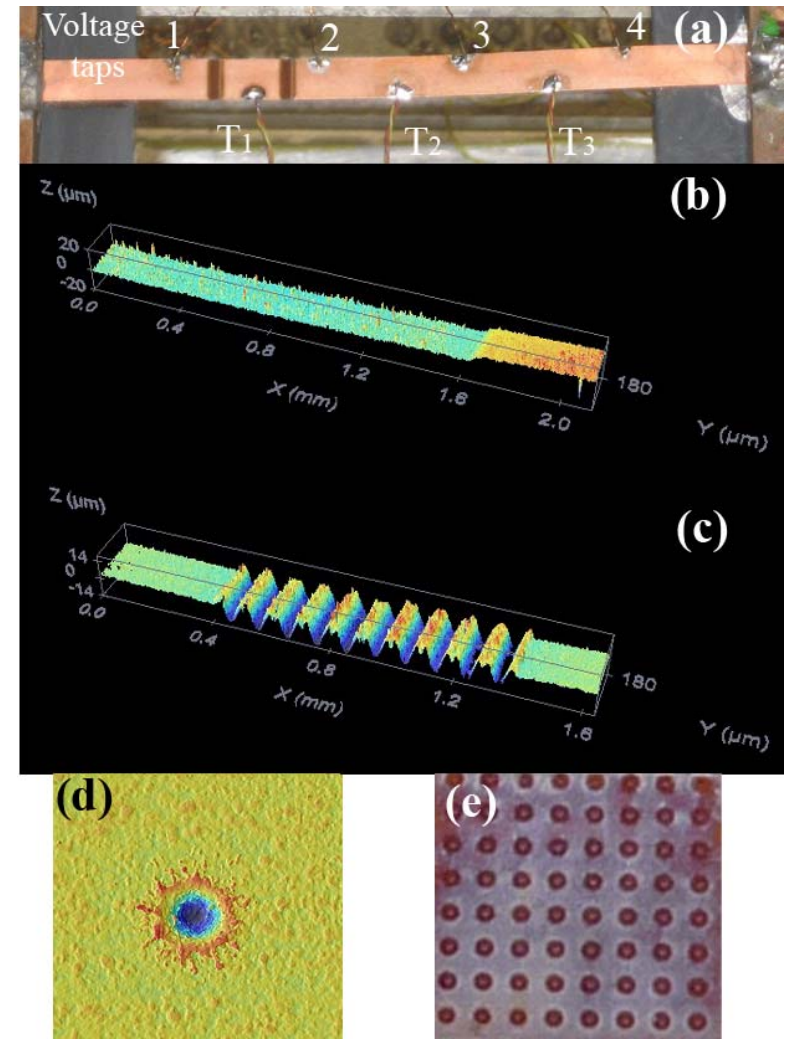

Fig. 1. (a) Geometry used in the fabrication of the defects with two sets at both sides of thermocouple $\mathrm{T}_{1}$. (b) $\mathrm{z}$-profile of the right half of the machined area in sample S1. (c) z-profile of the machined set of lines in sample S2. (d) Topography and (e) photograph showing the distribution of the set of holes in sample S3, distance between holes centers is $500 \mu \mathrm{m}$.

were chosen in order to assure that the laser ablation process only modifies the stabilizing layer without affecting the $I_{\mathrm{c}}(77 \mathrm{~K})$ values of the superconducting layer. The laser process can deteriorate the superconducting properties in two ways; either the depth of the induced defect reaches the superconducting layer in some points or the temperature increase that takes place during thermal laser ablation is too large. As it is shown in Fig. 1(a), defects were produced between voltage taps 1 and 2 and were repeated twice, one at each side of the thermocouple $\mathrm{T}_{1}$.

Three geometries of induced defects have been analyzed. In all cases, the topography of the machined regions was recorded using confocal microscopy ( $\mathrm{Pl} \mu-2300$ Sensofar) (Fig. 1(b) to (d)). In sample $\mathrm{S} 1$ two areas of $4 \times 3 \mathrm{~mm}^{2}$ were machined (Fig. 1(b)). The laser parameters used in the ablation process were: laser irradiance $6.5 \mathrm{GW} / \mathrm{cm}^{2}$, beam speed 100 $\mathrm{mm} / \mathrm{s}$, frequency $5 \mathrm{kHz}$ and distance between lines $0.007 \mathrm{~mm}$. In sample S2 (Fig. 1(a) and 1(c)), two sets of 10 transversal lines have been machined in a region of $1 \mathrm{~mm}$ on both sides of the thermocouple. The distance between the centers of the lines was $100 \mu \mathrm{m}$. This is the minimum distance that assures that the lines do not overlap. The laser irradiance, beam speed and frequency are the same as in sample S1. Finally, in sample S3 (Fig. 1(d) and (e)) two hole matrices have been machined. The distance between the centers holes was 500 $\mu \mathrm{m}$ and an area of $3.5 \times 3.5 \mathrm{~mm}^{2}$ was covered. In this case, the laser was working in a burst mode with an irradiance value of $8.4 \mathrm{GW} / \mathrm{cm}^{2}$.
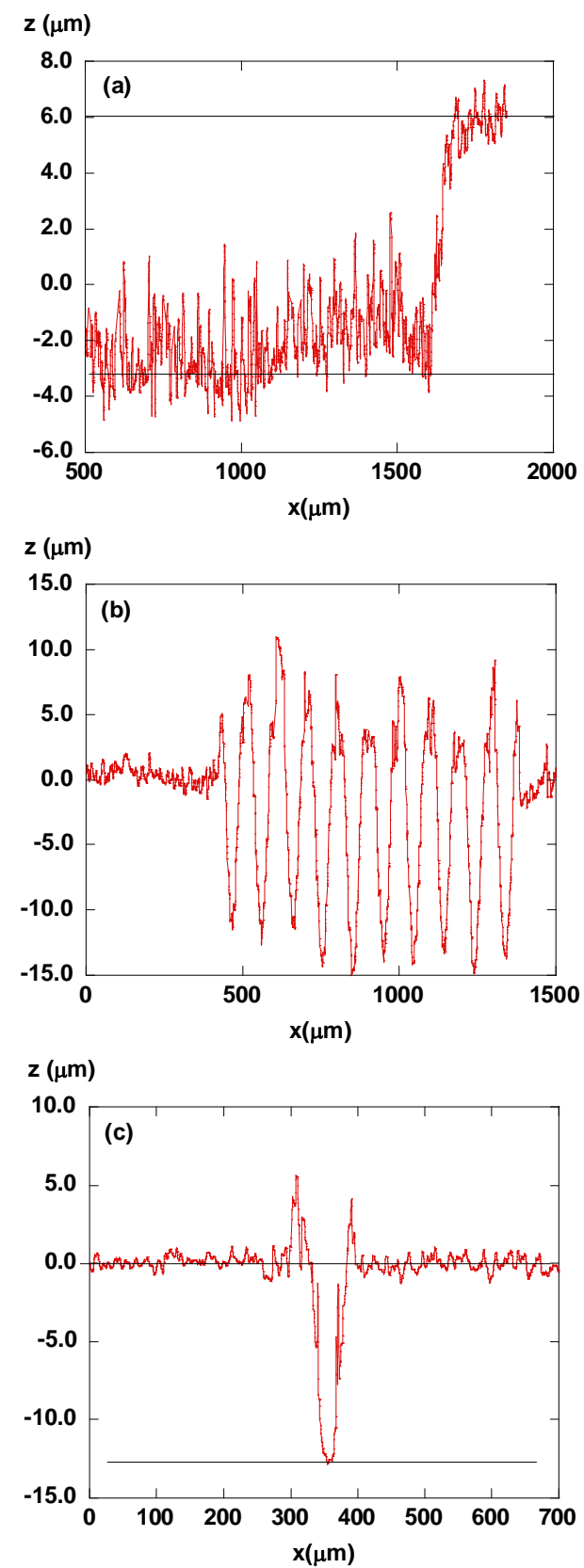

Fig. 2. z-profiles of the induced defects in the three samples: (a) Right half of the machined region in S1, (b) profile in a set of 10 lines in S2, (c) profile in any of the machined holes in S3.

The profiles of the different machined regions are presented in Fig. 2. In the case of sample S1, Fig 2(a) shows the profile in the right half of the machined region. Its depth is approximately $9.25 \mu \mathrm{m}$. From geometrical considerations it can be calculated that due to this thickness reduction the resistance of the $\mathrm{Cu}$ stabilizing layer should increase approximately $12 \%$. This value is very close to the experimental one in which an increase from $1.48 \mathrm{~m} \Omega$ to 1.64 $\mathrm{m} \Omega$ between voltage taps in the machined region was measured at room temperature.

In sample S2, the 10 lines are clearly observed (Fig. 2(b)). The depth of the lines ranges between 11.4 and $15 \mu \mathrm{m}$. Part of the ablated material is deposited in the regions between lines. 

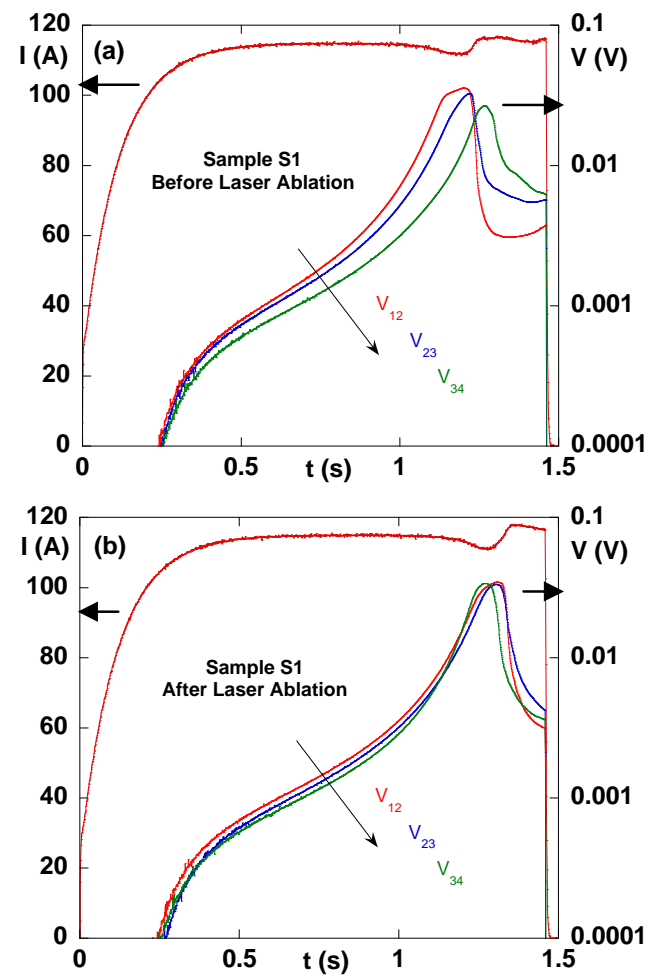

Fig. 3. Evolution of the voltage in the sample S1 during a pulse of $113 \mathrm{~A}$ for 1500 ms: (a) before and (b) after the laser ablation process.

By integrating this area it is possible to calculate that the total ablated volume is approximately $0.01 \mathrm{~mm}^{3}$ in each set of lines.

A typical profile of one of the holes machined in sample S3 is presented in Fig. 2(c). The hole has a conic shape with a diameter in the surface of $56 \mu \mathrm{m}$ and a typical depth of approximately $12.5 \mu \mathrm{m}$. Part of the ablated material can be observed around the machined hole (Fig. 1(d) and 2(c)).

After laser ablation, the $I-V$ characteristics at $77 \mathrm{~K}$ were recorded again. Measurements showed that the $I_{\mathrm{c}}(77 \mathrm{~K})$ values, defined using the $1 \mu \mathrm{V} / \mathrm{cm}$ criterion, remained unchanged, 84 A in the case of samples S1 and S3, and 97 A in sample S2. This confirmed that heat generated during ablation did not affect the superconducting layer.

In addition, the response to overcurrents was recorded again using electrical, thermal and Digital Speckle Phase Interferometry (DSPI) experiments.

\section{RESPONSE TO OVERCURRENTS}

The time evolution of the voltages measured between adjacent voltage taps was measured before and after the laser ablation process. The sample was immersed in liquid nitrogen. The observed phenomenology was the same in the three samples. Fig. 3 shows an example of the results obtained in the sample S1 during a $1500 \mathrm{~ms}$ pulse of $113 \mathrm{~A}$. This current value corresponds to $1.35 I_{\mathrm{c}}(77 \mathrm{~K})$ in this sample.

Before creating the defects (Fig. 3(a)), results show that the nucleate boiling regimen was reached with this current value. This is reflected in an increase of the cooling capability of the liquid nitrogen, a reduction of the temperature in the sample and, in consequence, in a reduction of the generated voltage. This change takes place in region $1-2(1.4 \mathrm{~cm}$ long) at $1.2 \mathrm{~s}$,
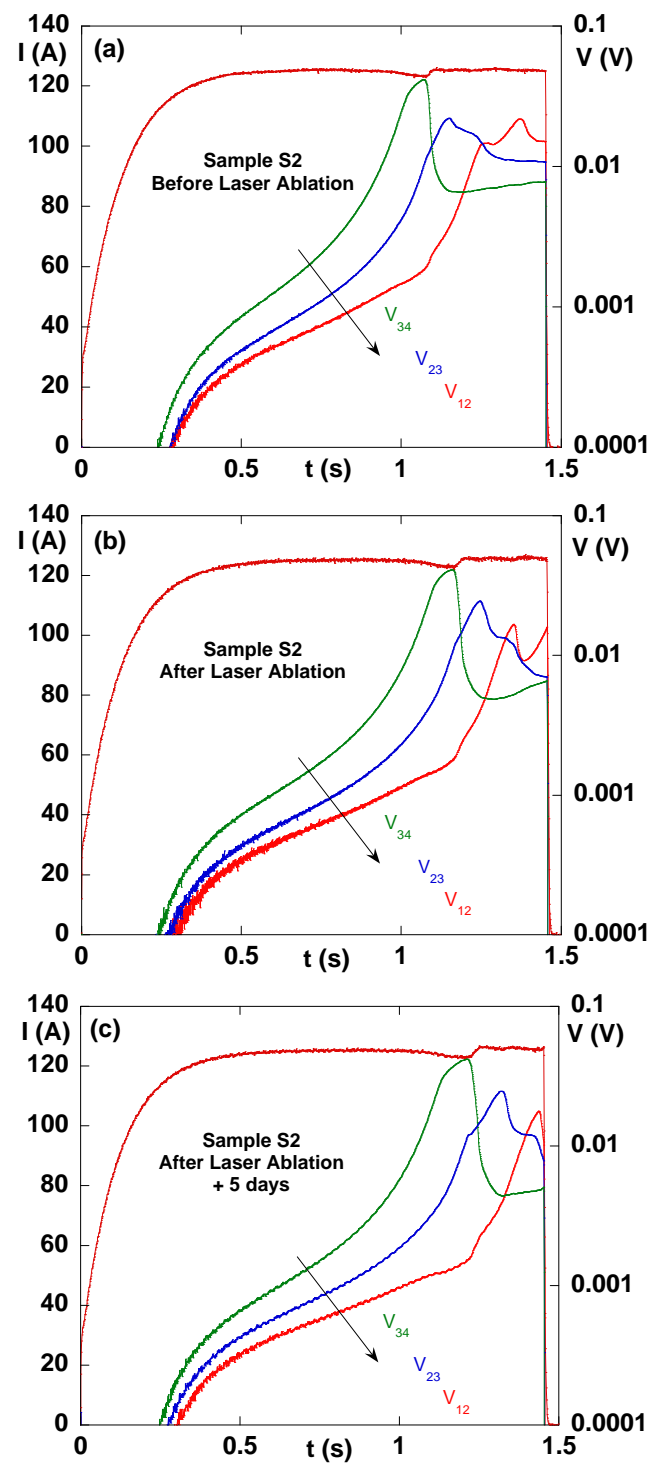

Fig. 4. Evolution of the voltage generation in the sample S2 during a pulse of 123 A for 1500 ms: (a) before, (b) after the laser ablation process and (c) 5 days after.

after having generated and electric field of $25 \mathrm{mV} / \mathrm{cm}$ and a temperature increase of $5.5 \mathrm{~K}$ in thermocouple 1 . This sudden decrease of $\Delta \mathrm{T}$ to $1.5 \mathrm{~K}$ and of electric field to values one order of magnitude lower is detected in region 2-3 at $1.25 \mathrm{~s}$ and in region 3-4 at $1.28 \mathrm{~s}$.

After the defects were induced in the $\mathrm{Cu}$ stabilizing layer, in region 1-2, the sample behavior shows the same trends (Fig. 3(b)). The nucleated boiling regime is observed when the same electric field value is reached. In this case this situation is reached at $1.33 \mathrm{~s}$. It seems that the sample behavior is more homogeneous in this case, but it is important to have in mind that in these experimental conditions, the sample response is strongly dependent on the status of liquid nitrogen, such as the level of liquid, the lapse of time between the liquid transfer to the cryostat and the experiment, or the disturbances created in previous measurements.

In order to confirm the possible influence of the situation of the liquid nitrogen around the sample in its response to 
overcurrents, in the case of sample S2 the measurements have been repeated several times during 18 days. Some examples are presented in Fig. 4, in the case of a pulse of 123 A, that corresponds to $1.23 I_{\mathrm{c}}(77 \mathrm{~K})$. In the original sample (Fig. 4(a)), voltage generation in region 3-4 is faster than in the other two regions. At $1.08 \mathrm{~s}, \mathrm{~V}_{34}$ shows the typical decrease associated with nucleate boiling. After this initial characterization, the two sets of lines were machined in region 1-2. As in the case of sample S1, the behavior is similar after laser ablation (Fig. 4(b)). Voltage and heat generation was slower in these conditions. Nucleate boiling is again reached in region 3-4, but in this case at $1.17 \mathrm{~s}$. These measurements have been repeated several times for 18 days, always performing the full measurement protocol. One example of the obtained measurements after 5 days is presented in Fig. 4(c). The observed phenomenology is always the same but with some changes among different set of experiments similar to those reported in the comparison of the measurements before and just after the laser ablation process. In this particular case, the nucleate boiling took place at $1.22 \mathrm{~s}$, but in other measurements values between 1.10 and 1.25 s were obtained.

Sample S3 shows the same behavior. In consequence, the defects that have been created in the stabilizing layer do not play an important role on how heat generation takes place in the sample when the current exceeds $I_{\mathrm{c}}$. Another important factor is that properties of the samples with defects do not deteriorate after several thermal cycles.

\section{DSPI OBSERVATIONS}

The same ablation process as performed in sample S1, with two areas of $4 \times 2 \mathrm{~mm}^{2}$, was performed in a second sample with $I_{\mathrm{c}}(77 \mathrm{~K})=97 \mathrm{~A}$ (Fig. 5(a)). In this case, characterization has been completed with DSPI observations. These measurements visualize the sample deformation associated with thermal expansion due to heat generated during the current pulse $[1,6$, 7]. As the sample was fixed at the two extremes, this deformation produces a sample bending with a maximum in the center.

DSPI produces a fringe map in which each fringe represents a deformation perpendicular to the sample surface of 0.27 $\mu \mathrm{m} /$ fringe. The sample situation has been recorded every 35 ms. As a consequence, if an image is compared with the previous one, sample deformation in the last $35 \mathrm{~ms}$ can be visualized. Two examples are presented in Fig. 5(b) and (c), before and after ablation, respectively. In these two cases, the sample was immersed in liquid nitrogen and a current pulse of $1.35 I_{\mathrm{C}}(77 \mathrm{~K})$ was applied. The electrical and thermal response of the sample is similar to the behavior presented in Fig. 3(a) for sample $\mathrm{S} 1$.

Fig. 5(b) shows the deformation map obtained in the original sample when $\mathrm{V}_{12}$ reached a value of $2.8 \mathrm{mV}$. The scale used in this figure is the same as in Fig. 5(a) in order to facilitate the comparison between the two images. The deformation pattern shows a maximum at the center of approximately 6.5 fringes, that means a perpendicular deformation of $1.75 \mu \mathrm{m}$ at this point. During the previous $35 \mathrm{~ms}, \mathrm{~V}_{12}$ increased by $0.5 \mathrm{mV}$.

This characterization was repeated again after having

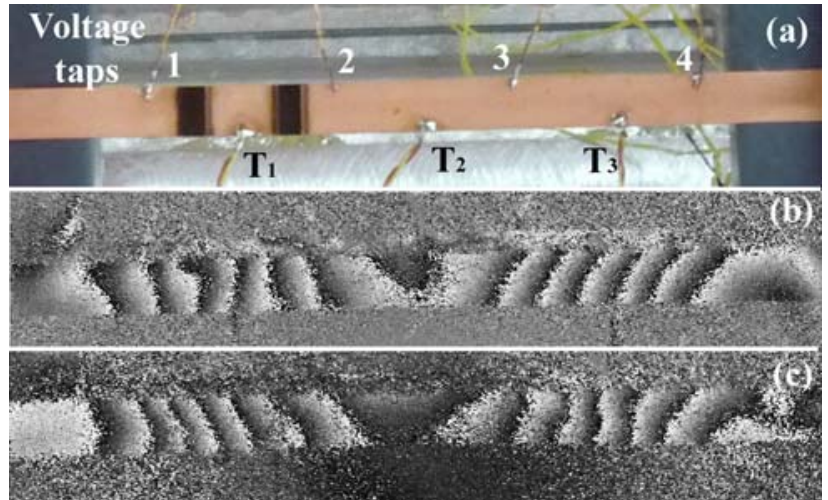

Fig. 5. (a) Photograph of the sample arrangement used in DSPI observations. Two areas of $4 \times 2 \mathrm{~mm}^{2}$ were machined in the sample. (b) DSPI maps in the original sample associated with the deformation in the last $35 \mathrm{~ms}$ when $\mathrm{V}_{12}=2.8 \mathrm{mV}$. (c) DSPI maps observed after laser ablation in similar experimental conditions.

produced the defects. As can be observed in Fig. 5(c), the sample with the defects shows a similar fringe pattern when heating conditions are similar, i.e., when $\mathrm{V}_{12}=2.8 \mathrm{mV}$ and $\Delta \mathrm{V}_{12}=0.5 \mathrm{mV}$. The defects produced in region $1-2$ do not modify the deformation maps associated with heating.

\section{CONCLUSIONS}

Several controlled defects have been introduced in the stabilizing layer of coated conductors using laser ablation without affecting the superconducting layer. The objective was to study the influence of these defects in the possible existence of inhomogeneities in the heat generation when a current higher than the critical current was applied.

Our results show that these controlled defects do not modify the response of the material to overcurrents, at least, when sample is immersed in liquid nitrogen.

\section{REFERENCES}

[1] L. A. Angurel, E. Martínez, F. Lera, S. Recuero, N. Andrés, M. P. Arroyo, Y.-Y. Xie, V. Selvamanickam, "Quench detection in $\mathrm{YBa}_{2} \mathrm{Cu}_{3} \mathrm{O}_{7-\delta}$ coated conductors using interferometric techniques”, Jour. Appl. Phys., vol. 104, 093916 (6p), 2008.

[2] X. Wang, U. P. Trociewitz, J. Schwartz, "Self-field quench behaviour of $\mathrm{YBa}_{2} \mathrm{Cu}_{3} \mathrm{O}_{7-\delta}$ coated conductors with different stabilizers”, Supercond. Sci. Technol., vol. 22, 085005 (13pp), 2009

[3] N. Y. Kwon, H. S. Kim, K. L. Kim, S. W. Yim, H.-R. Kim, O-B. Hyun, H.M. Kim, H.G. Lee, "Influence of stabilizer thickness on overcurrent test of YBCO-coated conductors”, Supercond. Sci. Technol., vol. 22, 045003 (6pp), 2009

[4] G. A. Levin, K. A. Novak, P. N. Barnes, “The effects of superconductorstabilizer interfacial resistance on the quench of a current-carrying coated conductor", Supercond. Sci. Technol., vol. 23, 014021 (8pp), 2010

[5] W.-K- Chan, P- J- Masson, C. A. Luongo, J. Schwartz, "Influence of Inter-Layer Contact Resistances on Quench Propagation in $\mathrm{YBa}_{2} \mathrm{Cu}_{3} \mathrm{O}_{7-\delta}$ Coated Conductors”, IEEE Trans. Appl. Supercond., vol. 19, no. 3, pp. 2490-2495, Jun. 2009.

[6] L. A. Angurel, E. Martínez, F. Lera, S. Recuero, N. Andrés, M. P. Arroyo, Y.-Y. Xie, V. Selvamanickam, "Analysis of Quench Initiation in YBCO Coated Conductors Using Optical Interferometric Techniques” IEEE Trans. Appl. Supercond., vol. 19, no. 3, pp. 3479-3482, Jun. 2009.

[7] L.A. Angurel, N. Andrés, M.P. Arroyo, S. Recuero, E. Martínez, J. Pelegrín, F. Lera, J.M. Andrés, “Application of Optical Techniques in the Characterization of Thermal Stability and Environmental Degradation in High Temperature Superconductors" in Superconductor, Ed. by Adir Moyses Luiz, 2010, ISBN: 978-953-307-107-7, Sciyo, pp. 179-202. 